\title{
Origin of the inverse spin-switch behavior in manganite/cuprate/manganite trilayers
}

\author{
N. M. Nemes* \\ Departamento de Fisica Aplicada III, GFMC, Universidad Complutense de Madrid, 28040 Madrid, Spain \\ and Instituto de Ciencia de Materiales de Madrid, Consejo Superior de Investigaciones Cientificas, 28049 Cantoblanco, Spain \\ M. García-Hernández \\ Instituto de Ciencia de Materiales de Madrid, Consejo Superior de Investigaciones Cientificas, 28049 Cantoblanco, Spain
}

S. G. E. te Velthuis and A. Hoffmann

Materials Science Division, Argonne National Laboratory, Argonne, Illinois 60439, USA

C. Visani, J. Garcia-Barriocanal, V. Peña, D. Arias, Z. Sefrioui, C. Leon, and J. Santamaría
Departamento de Fisica Aplicada III, GFMC, Universidad Complutense de Madrid, 28040 Madrid, Spain

(Received 6 August 2008; published 19 September 2008)

\begin{abstract}
We studied ferromagnet/superconductor/ferromagnet trilayers based on $\mathrm{La}_{0.7} \mathrm{Ca}_{0.3} \mathrm{MnO}_{3}$ manganite and $\mathrm{YBa}_{2} \mathrm{Cu}_{3} \mathrm{O}_{7-\delta}$ (YBCO) high- $T_{c}$ cuprate with magnetoresistance and magnetization measurements. We find an inverse superconducting spin-switch behavior, where superconductivity is favored for parallel alignment of the magnetization in the ferromagnetic layers. We argue that this inverse superconducting spin switch originates from the transmission of spin-polarized carriers into the superconductor. In this picture, the thickness dependence of the magnetoresistance yields the spin-diffusion length in YBCO as $13 \mathrm{~nm}$. A comparison of bilayers and trilayers allows ruling out the effect of the stray fields of the domain structure of the ferromagnet as the source of the inverse superconducting spin switch.
\end{abstract}

DOI: 10.1103/PhysRevB.78.094515

PACS number(s): 74.78.Fk, 72.25.-b, 75.47.-m

\section{INTRODUCTION}

Ferromagnetism and singlet superconductivity rarely coexist in the same material due to their antagonistic nature. The competition and interplay between these long-range ordering phenomena at the interface of artificial thin-film hybrids may couple the superconducting and ferromagnetic properties strongly. More specifically proximity effect or the stray fields of domains or domain walls (including spontaneous vortices) may be used to imprint the magnetic response of the ferromagnet into the superconductor allowing a fast and low dissipation detection of the magnetization switching. This provides an interesting avenue for the search of new concepts and phenomena in the development of spintronic devices. In proximity-coupled ferromagnet/superconductor/ ferromagnet $(\mathrm{F} / \mathrm{S} / \mathrm{F})$ structures with thin superconductors, the critical temperature is modulated by the relative orientation of the magnetization in the $\mathrm{F}$ layers ${ }^{1,2}$ (superconducting spin switch). A larger $T_{c}$ with antiparallel (AP) magnetizations compared to parallel $(\mathrm{P})$ magnetizations results from the averaging (compensation) of the exchange field over the coherent volume. These concepts have been experimentally realized in structures combining low- $T_{c}$ superconductors and transition-metal ferromagnets. ${ }^{3-5}$

Recent reports ${ }^{6-8}$ have shown an inverse superconducting spin-switch behavior in $\mathrm{F} / \mathrm{S} / \mathrm{F}$ structures with strong ferromagnets, where superconductivity is favored for parallel orientation of the adjacent magnetizations. Its origin is a subject of debate. Normal ${ }^{4}$ and inverse ${ }^{8,9}$ superconducting spin switches have been found by different groups in very similar $\mathrm{Ni} / \mathrm{Nb} / \mathrm{Ni}$ and $\mathrm{Py} / \mathrm{Nb} / \mathrm{Py}$ samples, highlighting the key role of the interface. Moreover, while some reports suggest an enhanced pair breaking by spin-polarized quasiparticles in the antiparallel configurations, others emphasize the effect of stray fields in depressing the superconductivity during the magnetization switching. ${ }^{10,11}$ The enhanced (perpendicular) stray field at Bloch-type domain walls in ferromagnets with in-plane magnetization may depress superconductivity if the coherence length $\left(\xi_{\mathrm{GL}}\right)$ is smaller than the domain-wall thickness $(\delta)$ or even nucleate vortices if the stray field is larger than the lower critical field $H_{c 1}^{\mathrm{S}}$ of the superconductor. ${ }^{7,12}$

In this paper we examine this issue in junctions based on manganites and cuprates where the interplay between ferromagnetism and superconductivity has recently shown a wealth of novel and interesting effects. ${ }^{13-15}$ This system is very amenable for this study since, on one hand, interfaces can be grown atomically flat to minimize the chance of roughness creating extrinsic stray fields and, on the other hand, the F/S proximity effect is expected to be absent owing to the high spin polarization of the ferromagnet ${ }^{16}$ and to the short $\xi_{\mathrm{GL}}$ of the superconductor. We find that $\mathrm{F} / \mathrm{S} / \mathrm{F}$ structures exhibit large magnetoresistance peaks for antiparallel alignment of the magnetization of the ferromagnetic layers, providing a clear signature of inverse superconducting spinswitch behavior. In contrast F/S bilayers exhibit clear evidence of nucleation of superconductivity at domain walls with no evidence for deterioration of the superconductivity due to stray fields. We argue that spin polarization is a key ingredient for the observation of the inverse superconducting spin-switch effect. By examining the dependence on the thickness of the superconducting layer, we obtain a measure of the spin-diffusion length in $\mathrm{YBa}_{2} \mathrm{Cu}_{3} \mathrm{O}_{7-\delta}(\mathrm{YBCO})$.

\section{EXPERIMENTAL DETAILS}

Epitaxial thin films were grown on (100) oriented $\mathrm{SrTiO}_{3}$ substrates using a high-pressure dc sputtering system using a 

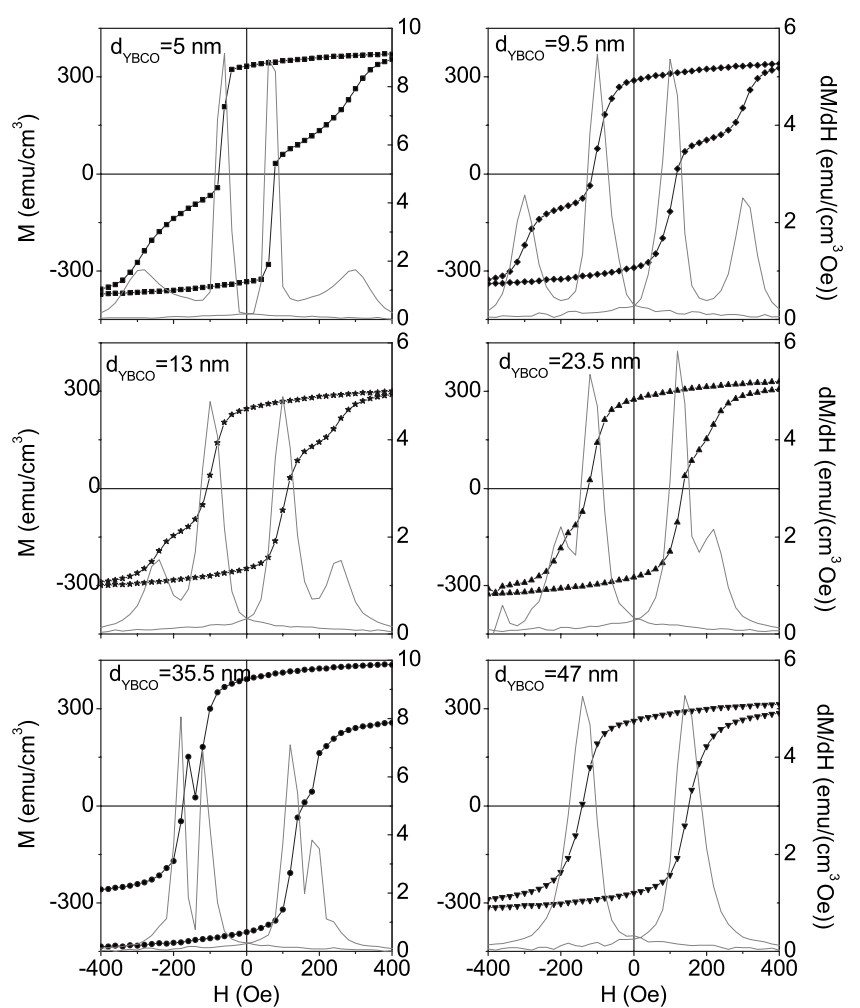

FIG. 1. Magnetization hysteresis loops (symbols) and their derivatives (thin lines) for LCMO/YBCO/LCMO trilayers with varying YBCO thickness between 5 and $48 \mathrm{~nm}$, below the superconducting transition, at $T=5 \mathrm{~K}$.

pure oxygen atmosphere (3.4 mbar) and a high growth temperature $\left(900{ }^{\circ} \mathrm{C}\right) .{ }^{17-19}$ Magnetization was measured with a $5 \mathrm{~T}$ superconducting quantum interference device (SQUID) magnetometer. Temperature-dependent magnetoresistance was measured in a cryostat equipped with a 9 T magnet using a current in-plane (CIP) geometry. All samples presented here have 15-nm-thick (40 unit cells) ferromagnetic $\mathrm{La}_{0.7} \mathrm{Ca}_{0.3} \mathrm{MnO}_{3}$ (LCMO) electrodes. The magnetic field was aligned within $0.05^{\circ}$ parallel to the substrate in the [010] direction of the substrate and perpendicular to the current.

\section{RESULTS}

In the following we focus on how the magnetoresistance due to antiparallel alignment changes with the thickness of the superconducting spacer. To this end, it is of primary importance to examine if the antiparallel alignment over a magnetic-field range is maintained over the whole thickness range. Hysteresis $[M(H)]$ loops measured below $(T=5 \mathrm{~K})$ and above the superconducting onset $(T=100 \mathrm{~K})$ display a clear plateau characteristic of antiparallel alignment as shown for a representative set of samples with varying YBCO spacer thickness in Figs. 1 and 2. Polarized neutron reflectivity shows that the positions of the peaks of the derivative of the $M(H)$ loops correspond to the switching fields of the individual ferromagnetic layers $\left(H_{c}^{\mathrm{F}}\right){ }^{6}$ Thus the respective coercive fields can be obtained from the two peaks of the derivative $(d M / d H)$ as displayed in Fig. 3. The region
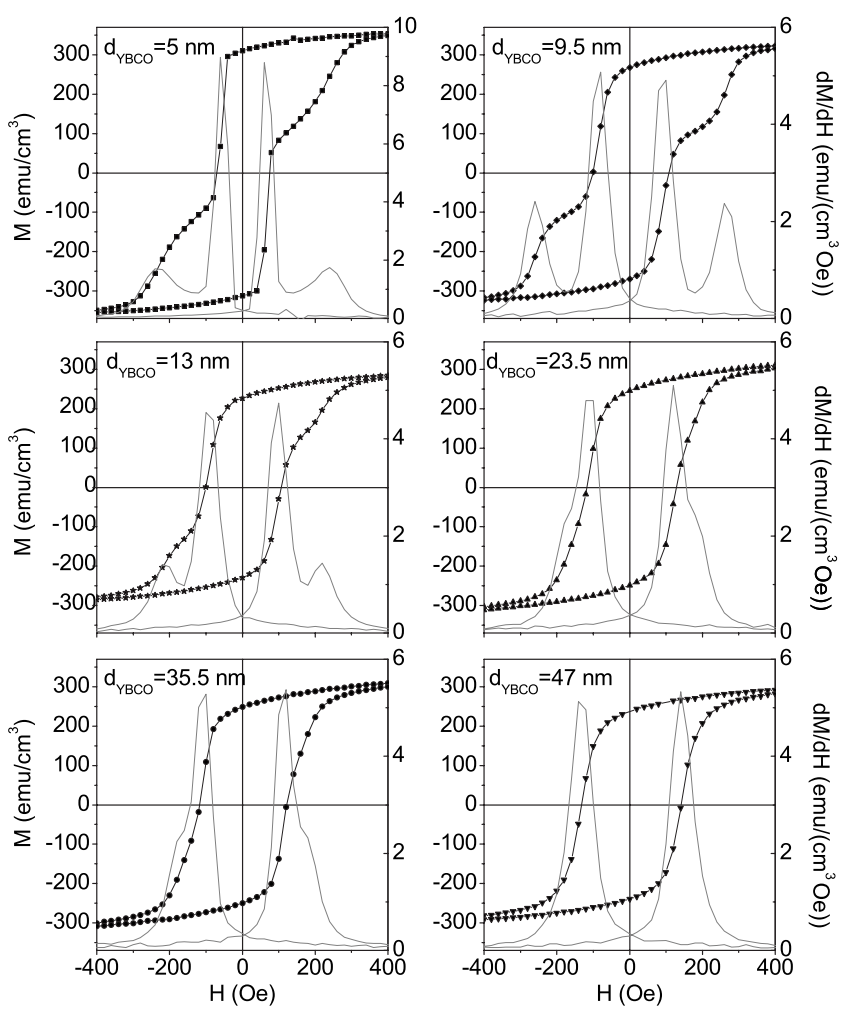

FIG. 2. Magnetization hysteresis loops (symbols) and their derivatives (thin lines) for $\mathrm{LCMO} / \mathrm{YBCO} / \mathrm{LCMO}$ trilayers with varying YBCO thickness between 5 and $48 \mathrm{~nm}$, above the superconducting transition, at $T=100 \mathrm{~K}$.

of antiparallel alignment shrinks rapidly with increasing YBCO spacer thickness. A weak shoulder on the main peak of $d M / d H$ can be seen up to a thickness of $35 \mathrm{~nm}$ in the data at $100 \mathrm{~K}$, while two distinct peaks are resolved for this sample at $5 \mathrm{~K}$, showing that antiparallel alignment persists up to this limit. On the contrary, the 47-nm-thick sample displays a unique coercive field even at $5 \mathrm{~K}$. Yet, the height of the magnetoresistance peak decreases apace with increasing spacer thickness as demonstrated below.

We have measured magnetoresistance at fixed temperatures along the superconducting transition while sweeping a magnetic field (between -1 and $+1 \mathrm{~T}$ ) applied parallel to the layers. The lower panel of Fig. 4 shows a typical $R(H)$ curve on a trilayer with 8-nm (7 unit cells)-thick YBCO measured at a resistance drop $R_{\min } / R_{n} \sim 10^{-4}$ relative to the normalstate $R_{n}$. Large magnetoresistance peaks can be seen far be-

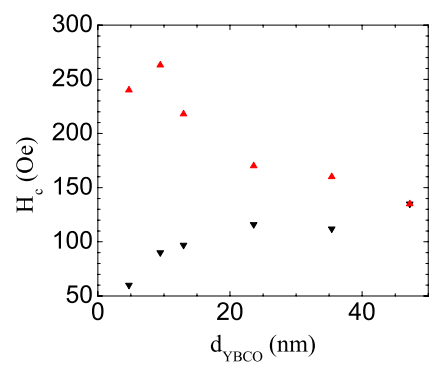

FIG. 3. (Color online) Coercive field vs YBCO spacer thickness of the trilayers at $T=100 \mathrm{~K}$ extracted from Fig. 2 . 


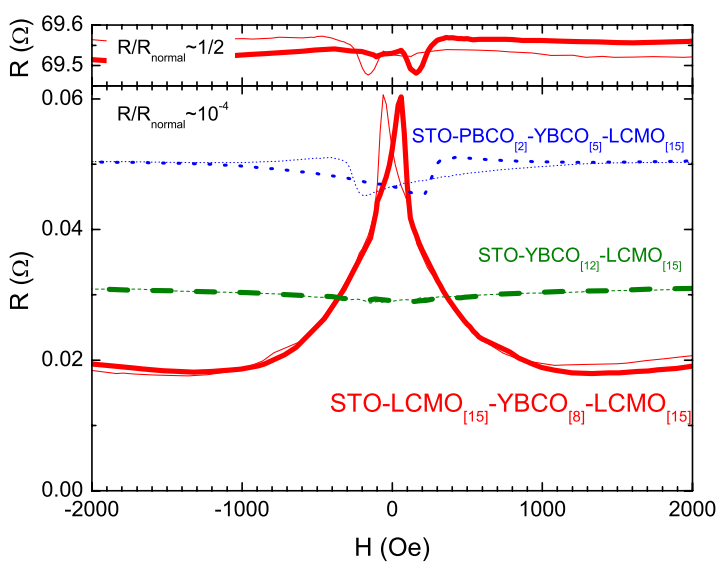

FIG. 4. (Color online) Upper panel: Magnetoresistance of a trilayer with $8 \mathrm{~nm}$ YBCO measured at $R_{\min } / R_{n} \sim 0.5$. Note the negative magnetoresistance peak. Lower panel: Magnetoresistance measured at a resistance drop $R_{\min } / R_{n} \sim 10^{-4}$. Continuous red line: Trilayer with $8 \mathrm{~nm}$ YBCO. Note the large positive magnetoresistance peak. Dashed green line: Bilayer with $12 \mathrm{~nm}$ YBCO. Note the lack of magnetoresistance features at low field. Blue dotted line: Bilayer with $5 \mathrm{~nm}$ YBCO. Note the negative magnetoresistance peak. Data are shown with thick lines for increasing fields with thin lines for decreasing fields.

low the onset and centered on a field interval where, owing to different coercivities, the magnetic moments of the ferromagnetic layers are antiparallel as determined by polarized neutron reflectivity (data not shown; see Ref. 6). Increasing temperature results in a decrease in the magnetoresistance. Near the onset, as depicted by the upper panel of Fig. 4 at transition midpoint, there appears a negative magnetoresistance peak centered at $H_{c}^{\mathrm{F}}$ of the top LCMO in this particular trilayer.

To examine whether magnetoresistance is related to stray fields from domain walls in the ferromagnet, which has been proposed as an explanation for positive magnetoresistance peaks, we have investigated related bilayer samples. Two examples are shown in Fig. 4. In the bilayer with a 12-nmthick YBCO bottom electrode there is no magnetoresistance feature at low field. The slowly rising background at higher fields, present in all superconducting films and strongly dependent on the alignment of film plane and magnetic field, is due to vortices (green dashed line). In the much thinner bilayer (with 5-nm-thick YBCO) a 2-nm-thick $\mathrm{PrBa}_{2} \mathrm{Cu}_{3} \mathrm{O}_{7}$ buffer was used to further improve the $\mathrm{YBCO}$ growth and thus the YBCO/LCMO interface quality (blue dotted line). ${ }^{20}$ This bilayer shows a well-defined negative magnetoresistance peak centered at $H_{c}^{\mathrm{F}}$ of the LCMO. Negative magnetoresistance peaks have been observed in F/S bilayers where the averaging out of the inhomogeneous stray field of the domain wall or of oppositely magnetized neighboring domains over the coherent volume promotes superconductivity nucleation at domain walls. ${ }^{21,22}$ It is important to remark that while trilayers show positive and very large magnetoresistance peaks, bilayers show no or very small negative peaks. These observations rule out the effect of stray fields as an origin of the observed magnetoresistance in this trilayer system, because trilayers and bilayers with equally thick YBCO
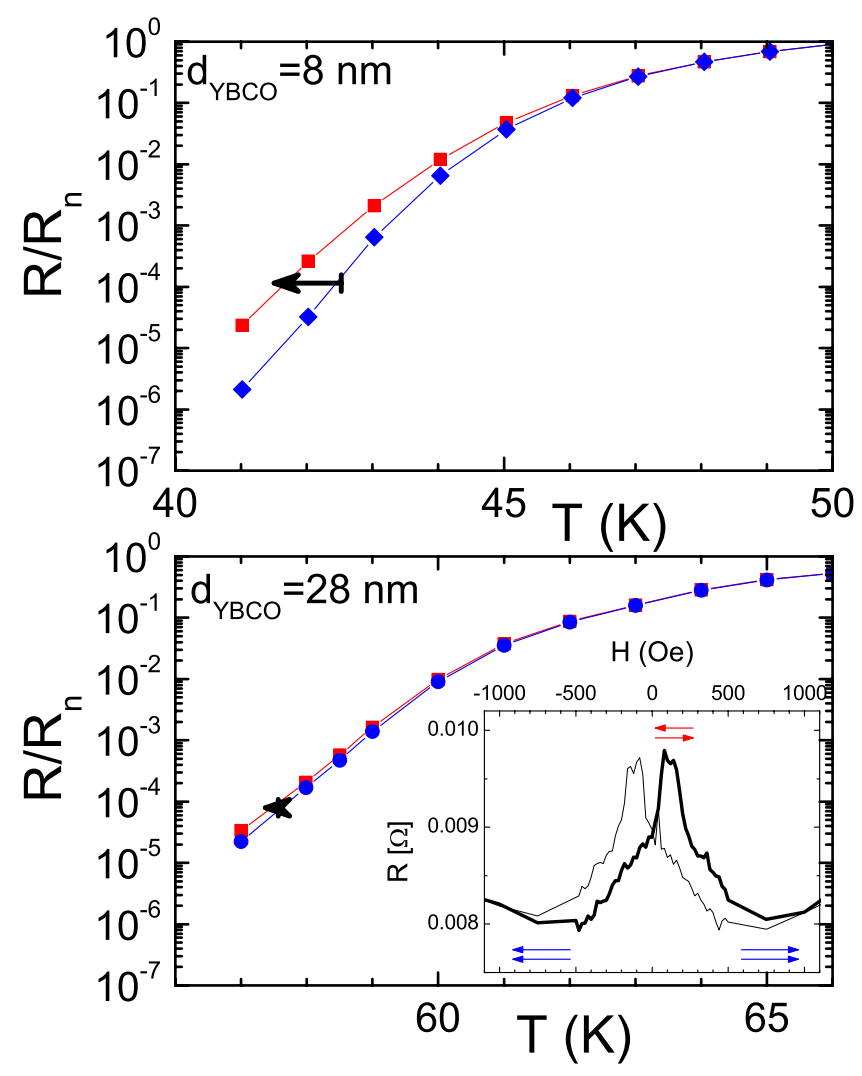

FIG. 5. (Color online) Trilayers with (upper panel) thin $(8 \mathrm{~nm})$ YBCO and (lower panel) thick $(28 \mathrm{~nm})$ YBCO. Modulation of the transition temperature, indicated by the arrow, obtained from the maximum (antiparallel, red squares) and minimum (parallel, blue circles) resistances. Inset of lower panel: Typical magnetoresistance in increasing (thick) and decreasing (thin) fields at $R_{\min } / R_{n} \sim 10^{-4}$. Magnetoresistance corresponding to the upper panel is shown in Fig. 4.

and LCMO and having similar interface quality shall have comparable stray fields.

We need to compare trilayers with different YBCO thickness, but $T_{c}$ is progressively suppressed and the transition width is increased in thinner YBCO films. ${ }^{18}$ Therefore, we calculated the increase in the width of the superconducting transition $\Delta T_{c}^{\mathrm{P} \mapsto \mathrm{AP}}$ caused by the change in the relative orientation of the magnetization of the two ferromagnetic layers. The measured resistance was normalized by the normalstate resistance $R_{n}$ just at the onset, which is a good proxy to scaling by the YBCO thickness as the samples have identical LCMO layers and lateral dimensions. Resistance values at the maxima $\left(R_{\max }\right)$ and minima $\left(R_{\min }\right)$ were used to construct resistance vs temperature curves, $T^{\mathrm{AP}}(R)$ and $T^{\mathrm{P}}(R)$, in the antiparallel and parallel configurations of the F layers (Fig. 5). Magnetoresistance at a given temperature is related, through the slope of $R(T)$, to the local increase in the superconducting transition width defined as $\Delta T_{c}^{\mathrm{P} \mapsto \mathrm{AP}}(R)=T^{\mathrm{P}}(R)$ $-T^{\mathrm{AP}}(R)$. Thus, we converted our $T$-dependent magnetoresistance sweeps into $R$-dependent $\Delta T_{c}^{\mathrm{P} \mapsto \mathrm{AP}}$, indicated by the horizontal black arrows in Fig. 5, which is the chosen quantity in many of the studies of conventional superconducting spin switches. ${ }^{4,8,10}$ 

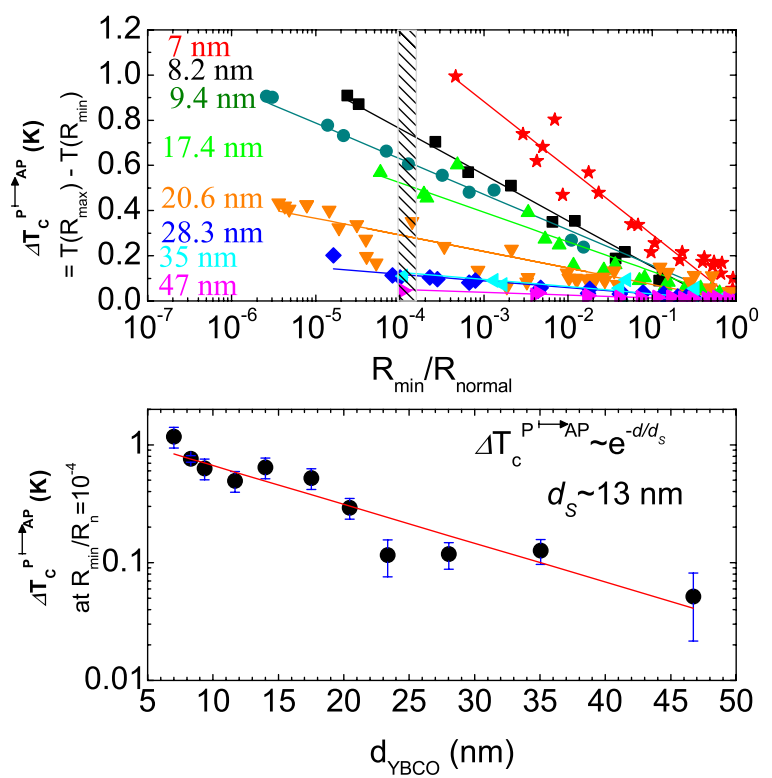

FIG. 6. (Color online) Upper panel: $\Delta T_{c}^{\mathrm{P} \mapsto \mathrm{AP}}$, as defined in Fig. 5 , decreases linearly with the logarithm of the resistance of the superconductor below $T_{c}$. The lines are logarithmic linear fits. The YBCO spacer thicknesses are given on the left. Lower panel: Decreasing pair-breaking effect with increasing YBCO thickness quantified by taking $\Delta T_{c}^{\mathrm{P} \mapsto \mathrm{AP}}\left(R_{\min } / R_{n}=10^{-4}\right)$. The line is an exponential fit.

Samples with different YBCO thickness may be compared by picking the value of $\Delta T_{c}^{\mathrm{P} \rightarrow \mathrm{AP}}$ at a given $R_{\min } / R_{n}$. We chose $R_{\min } / R_{n}=10^{-4}$, as shown by the gray bar in the upper panel of Fig. 6. The effect is large. For the thinnest samples parallel and antiparallel resistance curves are separated by more than $1 \mathrm{~K}$. This is in contrast to recent reports on proximity-coupled structures where $T_{c}$ shifts by anywhere between 2 and $40 \mathrm{mK} \cdot{ }^{3,4,7,10,11} \mathrm{We}$ observed an exponential decay of $\Delta T_{c}^{\mathrm{P} \mapsto \mathrm{AP}}$ with increasing thickness of the superconducting spacer (Fig. 6, lower panel). The slope indicates a characteristic length scale of $13 \mathrm{~nm}$, which can be related to the spin-diffusion length in YBCO. A different $R_{\min } / R_{n}$ choice would simply shift the data in the lower panel of Fig. 6 , with no effect on its slope on a logarithmic scale.

\section{DISCUSSION}

To understand the magnetoresistance we propose a mechanism involving a different effective density of quasiparticles, which depends on the relative orientation of the magnetization in the ferromagnetic layers. Let us consider a normal/superconducting interface with no applied voltage; then a dynamical equilibrium exists in which particles approaching the interface from the normal side may diffuse into the superconductor and quasiparticles of the superconductor are transmitted into the normal metal at the same rate. ${ }^{23}$ In $\mathrm{F} / \mathrm{S} / \mathrm{F}$ double junctions, with thin superconducting layers, the simultaneous equilibrium of the two interfaces requires that quasiparticles thermally excited above the gap and transmitted through one interface may escape through the other and vice versa, since at equilibrium there should be no net charge transport. There will be a sizeable fraction $k_{B} T / \Delta(T)$ of quasiparticles with energies larger than the gap $\Delta(T)$ that may be transmitted into the superconductor since close to $T_{c}$ the gap approaches zero. A mechanism of $\mathrm{F} / \mathrm{S} / \mathrm{F}$ transport of subgap energy spins based on Andreev reflection ${ }^{24}$ can be ruled out in the present case since Andreev reflection will be suppressed at the interface given the high spin polarization of LCMO.

In particular, for highly spin-polarized ferromagnets, the situation depends on the relative alignment of the magnetization in the F layers. For parallel alignment, electrons transmitted from one electrode can escape through the opposing interface provided the superconductor is thinner than the spin-diffusion length. However, in the antiparallel configuration, electrons transmitted from one ferromagnetic electrode will be reflected if the superconducting spacer is thinner than the spin-diffusion length, as there are no states available at the Fermi level with the right spin orientation in a half metal. ${ }^{25}$ This causes an effective increase in the number of quasiparticles that self-consistently reduces $T_{c}$ due to a nonequilibrium process in the quasiparticle excitation spectrum. The excess quasiparticles may persist over large distances from the interface before they relax into Cooper pairs in the current to supercurrent conversion process, since the quasiparticle relaxation time diverges as $\tau_{Q} \sim 10^{-10} \mathrm{~s} /[\Delta(T) / \Delta(0)]$ close to $T_{c}{ }^{26}$ In addition, the $d$-wave pairing symmetry of YBCO allows introducing spinpolarized quasiparticle excitations at zero energy cost along nodal regions, thus increasing the importance of transmitted quasiparticles.

This process involves an increased quasiparticle density due to mirrorlike interfaces for the antiparallel alignment, yet it does not involve net spin or charge transport between the electrodes (CIP transport). When the thickness of the superconductor is larger than the spin-diffusion length, electrons transmitted from one electrode lose memory of their spin orientation and behave identically at the other interface irrespective of the magnetic configurations. This is consistent with the much smaller $T_{c}$ shift for 28-nm-thick YBCO as compared to 8-nm-thick YBCO (see Fig. 5). Furthermore, within this picture we may interpret the slope of the thickness dependence of $\Delta T_{c}^{\mathrm{P} \mapsto \mathrm{AP}}$ as a measure of the spindiffusion length. A logarithmic linear fit (lower panel of Fig. 6) yields for the spin-diffusion length $d_{s}=13 \mathrm{~nm}$ in YBCO. This value is in good agreement with previous estimates based on the thickness dependence of $T_{c}$ for YBCO in bilayer samples. ${ }^{27}$

Therefore, inverse and conventional superconducting spin switches in $\mathrm{F} / \mathrm{S} / \mathrm{F}$ junctions result from different mechanisms, namely, proximity effect and quasiparticle diffusion. These may be present simultaneously and compete to some extent in the same sample. Large spin polarization of the ferromagnetic electrodes or increasing the thickness of the superconductor beyond $\xi_{\mathrm{GL}}$ reduces proximity coupling and favors the observation of quasiparticle scattering at the F/S interface responsible for the inverse superconducting spinswitch behavior. Finally, an important issue is the interface transparency and the strength of the interface barrier. These determine the ratio of particles being transmitted versus those being Andreev reflected. In turn, this determines the 
operation of the $\mathrm{F} / \mathrm{S} / \mathrm{F}$ junction as conventional or inverse superconducting spin switch. This should be the reason why apparently similar structures with different interface properties yield opposite behaviors. $4,7,8,10,11$

\section{CONCLUSIONS}

In summary, we have shown that F/S/F structures based on cuprates and manganites exhibit an inverse superconducting spin-switch behavior, where superconductivity is favored for parallel magnetizations of the two ferromagnetic layers. The inverse superconducting spin switch is controlled by scattering (reflection) of spin-polarized quasiparticles at the F/S interface, which is strongly enhanced for antiparallel alignment of the $\mathrm{F}$ layers. The high spin polarization of the manganite and the $d$-wave superconductivity of the YBCO are crucial for observing this effect. This is a unique form of coupling between the superconducting properties and the magnetic state of the ferromagnetic electrodes that occurs when the thickness of the superconducting layer is shorter than the spin-diffusion length, and it may form the basis of novel device concepts for spintronics.

\section{ACKNOWLEDGMENTS}

This work was supported by MAT 2005 under Contract No. 06024 C02 01-02, the Joint US-Spain World Materials Proposal (NSF MWN Proposal DMR Contract No. 0709584 and MEC MAT 2007 Contract No. 30922-E), and by the US-DOE BES under Contract No. DE-AC02-06CH11357. N.M.N. acknowledges the "Ramon y Cajal" contract of the MICINN.

*nmnemes@fis.ucm.es

${ }^{1}$ A. I. Buzdin, Rev. Mod. Phys. 77, 935 (2005).

${ }^{2}$ F. S. Bergeret, A. F. Volkov, and K. B. Efetov, Rev. Mod. Phys. 77, 1321 (2005).

${ }^{3}$ J. Y. Gu, C. Y. You, J. S. Jiang, J. Pearson, Y. B. Bazaliy, and S. D. Bader, Phys. Rev. Lett. 89, 267001 (2002).

${ }_{4}^{4}$ I. C. Moraru, W. P. Pratt, and N. O. Birge, Phys. Rev. Lett. 96, 037004 (2006).

${ }^{5}$ G. X. Miao, K. S. Yoon, T. S. Santos, and J. S. Moodera, Phys. Rev. Lett. 98, 267001 (2007).

${ }^{6}$ V. Pena, Z. Sefrioui, D. Arias, C. Leon, J. Santamaria, J. L. Martinez, S. G. E. te Velthuis, and A. Hoffmann, Phys. Rev. Lett. 94, 057002 (2005).

${ }^{7}$ A. Y. Rusanov, S. Habraken, and J. Aarts, Phys. Rev. B 73, 060505(R) (2006).

${ }^{8}$ A. Singh, C. Surgers, and H. v. Lohneysen, Phys. Rev. B 75, 024513 (2007).

${ }^{9}$ J. Aarts and A. Y. Rusanov, C. R. Phys. 7, 99 (2006).

${ }^{10}$ R. Steiner and P. Ziemann, Phys. Rev. B 74, 094504 (2006).

${ }^{11}$ D. Stamopoulos, E. Manios, and M. Pissas, Phys. Rev. B 75, 014501 (2007).

${ }^{12}$ C. Bell, S. Tursucu, and J. Aarts, Phys. Rev. B 74, 214520 (2006).

${ }^{13}$ A. Pimenov, A. Loidl, P. Przyslupski, and B. Dabrowski, Phys. Rev. Lett. 95, 247009 (2005).

${ }^{14}$ J. Chakhalian et al., Nat. Phys. 2, 244 (2006).

${ }^{15}$ V. A. Vas'ko, V. A. Larkin, P. A. Kraus, K. R. Nikolaev, D. E. Grupp, C. A. Nordman, and A. M. Goldman, Phys. Rev. Lett.

78, 1134 (1997).

${ }^{16}$ M. J. M. de Jong and C. W. J. Beenakker, Phys. Rev. Lett. 74, 1657 (1995).

${ }^{17}$ Z. Sefrioui, M. Varela, V. Pena, D. Arias, C. Leon, J. Santamaria, J. E. Villegas, J. L. Martinez, W. Saldarriaga, and P. Prieto, Appl. Phys. Lett. 81, 4568 (2002).

${ }^{18}$ Z. Sefrioui, D. Arias, V. Pena, J. E. Villegas, M. Varela, P. Prieto, C. Leon, J. L. Martinez, and J. Santamaria, Phys. Rev. B 67, 214511 (2003)

${ }^{19}$ V. Pena, Z. Sefrioui, D. Arias, C. Leon, J. Santamaria, M. Varela, S. J. Pennycook, and J. L. Martinez, Phys. Rev. B 69, 224502 (2004).

${ }^{20}$ V. Pena, C. Visani, J. Garcia-Barriocanal, D. Arias, Z. Sefrioui, C. Leon, J. Santamaria, and C. A. Almasan, Phys. Rev. B 73, 104513 (2006).

${ }^{21}$ A. Y. Rusanov, M. Hesselberth, J. Aarts, and A. I. Buzdin, Phys. Rev. Lett. 93, 057002 (2004).

${ }^{22}$ Z. R. Yang, M. Lange, A. Volodin, R. Szymczak, and V. V. Moshchalkov, Nat. Mater. 3, 793 (2004).

${ }^{23}$ G. E. Blonder, M. Tinkham, and T. M. Klapwijk, Phys. Rev. B 25, 4515 (1982).

${ }^{24}$ J. Y. Gu, J. A. Caballero, R. D. Slater, R. Loloee, and W. P. Pratt, Phys. Rev. B 66, 140507(R) (2002).

${ }^{25}$ S. Takahashi, H. Imamura, and S. Maekawa, Phys. Rev. Lett. 82, 3911 (1999).

${ }^{26}$ M. Tinkham and J. Clarke, Phys. Rev. Lett. 28, 1366 (1972).

${ }^{27}$ S. Soltan, J. Albrecht, and H. U. Habermeier, Phys. Rev. B 70, 144517 (2004). 